\title{
Correction to: Breed susceptibility for common surgically treated orthopaedic diseases in 12 dog breeds
}

\author{
Gudrun Seeberg Boge ${ }^{1 *} \mathbb{E}$, Elena Regine Moldal ${ }^{1}$, Maria Dimopoulou$^{2}$, Eystein Skjerve ${ }^{3}$ and Annika Bergström²
}

\section{Correction to: Acta Vet Scand (2019) 61:19} https://doi.org/10.1186/s13028-019-0454-4

After publication of our article [1] we were notified that due to an error in the excel formula used to summarise the control population, the ID-registry data from the Swedish county Gävleborg was not included in the calculations. When including Gävleborg, as intended, the numbers in the adjusted Swedish control population change slightly. It does not influence the Norwegian control population.
The correct Tables 4 and 5, as well as the correct version of Additional file 1 are included in this correction.

In Table 4, the modified values are found in the Control population header, under the Sweden and Combined columns. In Table 5, the SLU and Combined columns contain the modified values.

*Correspondence: gudrun.seeberg.boge@nmbu.no

1 Department of Companion Animal Clinical Sciences, Faculty

of Veterinary Medicine, Norwegian University of Life Sciences, P.O. Box 369 sentrum, 0102 Oslo, Norway

Full list of author information is available at the end of the article

(c) The Author(s) 2020. This article is licensed under a Creative Commons Attribution 4.0 International License, which permits use, sharing, adaptation, distribution and reproduction in any medium or format, as long as you give appropriate credit to the original author(s) and the source, provide a link to the Creative Commons licence, and indicate if changes were made. The images or other third party material in this article are included in the article's Creative Commons licence, unless indicated otherwise in a credit line to the material. If material is not included in the article's Creative Commons licence and your intended use is not permitted by statutory regulation or exceeds the permitted use, you will need to obtain permission directly from the copyright holder. To view a copy of this licence, visit http://creativecommons.org/licenses/by/4.0/. The Creative Commons Public Domain Dedication waiver (http://creativecommons.org/publicdomain/zero/1.0/) applies to the data made available in this article, unless otherwise stated in a credit line to the data. 
Table 4 Breed distribution of dogs surgically treated for orthopaedic diseases and a geographically adjusted control group

\begin{tabular}{|c|c|c|c|c|c|c|}
\hline \multirow[t]{3}{*}{ Breed } & \multicolumn{3}{|c|}{ Eligible cases } & \multicolumn{3}{|c|}{ Control population } \\
\hline & Sweden & Norway & Combined & Sweden & Norway & Combined \\
\hline & N (\%) & $\mathrm{N}(\%)$ & N (\%) & $\mathrm{N}(\%)$ & $\mathrm{N}(\%)$ & N (\%) \\
\hline Mixed-breed & $114(44.9)$ & $53(30.1)$ & $167(38.8)$ & $3146(28.0)$ & 4359 (37.4) & $7505(32.5)$ \\
\hline Border collie & $8(3.2)$ & $5(2.8)$ & $13(3.0)$ & $331(3.0)$ & $425(3.7)$ & $756(3.2)$ \\
\hline CKCS & $5(2.0)$ & $9(5.1)$ & $14(3.3)$ & $556(5.0)$ & $556(4.8)$ & $1112(4.8)$ \\
\hline Chihuahua & $14(5.5)$ & $18(10.2)$ & $32(7.4)$ & $1113(10.0)$ & 985 (8.5) & $2099(9.0)$ \\
\hline English cocker spaniel & $1(0.4)$ & $3(1.7)$ & $4(0.9)$ & $547(4.9)$ & $539(4.6)$ & $1085(4.7)$ \\
\hline Flat-coated retriever & $3(1.2)$ & $4(2.3)$ & $7(1.6)$ & $479(4.3)$ & $356(3.1)$ & $835(3.5)$ \\
\hline German shepherd dog & $12(4.7)$ & $7(4.0)$ & $19(4.4)$ & $1125(10.0)$ & $591(5.1)$ & $1716(7.3)$ \\
\hline Golden retriever & $17(6.7)$ & $9(5.1)$ & $26(6.1)$ & $1080(9.6)$ & $754(6.5)$ & $1834(7.8)$ \\
\hline Jack Russel terrier & $9(3.5)$ & $6(3.4)$ & $15(3.5)$ & $457(4.1)$ & $710(6.1)$ & $1168(5.1)$ \\
\hline Labrador retriever & $32(12.6)$ & $20(11.4)$ & $52(12.1)$ & $1345(12.0)$ & $823(7.1)$ & $2168(9.3)$ \\
\hline Rottweiler & $19(7.5)$ & $22(12.5)$ & $41(9.5)$ & $540(4.8)$ & $469(4.0)$ & $1009(4.3)$ \\
\hline Shetland sheepdog & $8(3.2)$ & $7(4.0)$ & $15(3.5)$ & $506(4.5)$ & $319(2.7)$ & $825(3.5)$ \\
\hline Staff. bull terrier & $12(5.8)$ & $13(7.4)$ & $25(5.8)$ & $418(3.7)$ & $763(6.6)$ & $1181(5.1)$ \\
\hline Total (BuS)* & $254(51.2)$ & $176(36.1)$ & $430(43.7)$ & $11,226(100.0)$ & $10,886(100.0)$ & $22,112(100.0)$ \\
\hline Other breeds* & $242(48.8)$ & $312(63.9)$ & $554(56.3)$ & & & \\
\hline Total & $496(100.0)$ & $488(100.0)$ & $984(100.0)$ & & & \\
\hline
\end{tabular}

Control population calculated from registration numbers of each breed in the national ID-registries adjusted to reflect the source population of dogs surgically treated for orthopaedic diseases at the University Animal Hospital, Swedish University of Agricultural Sciences and the University Animal Hospital, Norwegian University of Life Sciences over a 5-year period

BuS breeds under study, CKCS Cavalier king Charles spaniel

*Data presented as number of dogs (percentage) of breeds under study

Table 5 Results from the logistic regression analyses of breed susceptibility for four common orthopaedic diseases in dogs

\begin{tabular}{|c|c|c|c|c|c|c|c|c|c|c|c|c|}
\hline \multicolumn{13}{|l|}{ a) Elbow dysplasia } \\
\hline \multirow[t]{2}{*}{ Breed } & \multicolumn{4}{|l|}{ SLU } & \multicolumn{4}{|l|}{ NMBU } & \multicolumn{4}{|l|}{ Combined } \\
\hline & N (\%) & OR & $95 \% \mathrm{Cl}$ & $\mathbf{P}$ & N (\%) & OR & $95 \% \mathrm{Cl}$ & $\mathbf{P}$ & N (\%) & OR & $95 \% \mathrm{Cl}$ & $P$ \\
\hline Mixed-breed & $13(22.0)$ & 1.00 & Ref. & - & $2(7.41)$ & 1.00 & Ref. & - & $15(17.4)$ & 1.00 & Ref. & - \\
\hline Flat-coated retriever & $0(0.0)$ & - & - & - & $2(7.41)$ & 12.24 & $(1.72-87.18)$ & 0.012 & $2(2.3)$ & 1.10 & $(0.25-4.83)$ & 0.899 \\
\hline German shepherd dog & $10(17.0)$ & 2.15 & $(0.94-4.92)$ & 0.070 & $3(11.11)$ & 11.06 & $(1.84-66.35)$ & 0.009 & $13(15.1)$ & 3.33 & $(1.57-7.62)$ & 0.002 \\
\hline Golden retriever & $7(11.9)$ & 1.57 & $(0.62-3.94)$ & 0.338 & $2(7.41)$ & 5.78 & $(0.81-41.10)$ & 0.080 & $9(10.5)$ & 2.23 & $(0.97-5.13)$ & 0.058 \\
\hline Labrador retriever & $19(32.2)$ & 3.42 & $(1.68-6.94)$ & 0.001 & $9(33.33)$ & 23.83 & $(5.14-110.51)$ & $<0.001$ & $28(32.6)$ & 5.79 & $(3.07-10.91)$ & $<0.001$ \\
\hline Rottweiler & $4(6.8)$ & 1.79 & $(0.58-5.52)$ & 0.309 & $8(29.63)$ & 37.18 & $(7.87-175.58)$ & $<0.001$ & $12(14.0)$ & 5.59 & (2.60-11.99) & $<0.001$ \\
\hline Staff. bull terrier & $6(10.2)$ & 2.80 & $(1.06-7.41)$ & 0.038 & $1(3.7)$ & 2.86 & $(0.25-31.54)$ & 0.392 & $7(8.1)$ & 2.75 & $(1.12-6.77)$ & 0.027 \\
\hline SLU & $59(68.9)$ & & & & & & & & $59(45.0)$ & 1.00 & Ref. & - \\
\hline NMBU & & & & & $27(60.0)$ & & & & 27 (20.6) & 0.56 & $(0.35-0.90)$ & 0.015 \\
\hline Other breeds & 27 (31.1) & & & & $18(40.0)$ & & & & $45(34.4)$ & & & \\
\hline Total & $86(100.0)$ & & & & $45(100.0)$ & & & & $131(100.0)$ & & & \\
\hline
\end{tabular}


Table 5 (continued)

\section{b) Cranial cruciate ligament disease}

\begin{tabular}{|c|c|c|c|c|c|c|c|c|c|c|c|c|}
\hline \multirow[t]{2}{*}{ Breed } & \multicolumn{4}{|l|}{ SLU } & \multicolumn{4}{|l|}{ NMBU } & \multicolumn{4}{|l|}{ Combined } \\
\hline & N (\%) & OR & $95 \% \mathrm{Cl}$ & $\mathbf{P}$ & N (\%) & OR & $95 \% \mathrm{Cl}$ & $\mathbf{P}$ & N (\%) & OR & $95 \% \mathrm{Cl}$ & $P$ \\
\hline Mixed-breed & $32(44.4)$ & 1.00 & Ref. & - & $13(26.0)$ & 1.00 & Ref. & - & $45(36.9)$ & 1.00 & Ref. & - \\
\hline Border collie & $0(0.0)$ & - & - & - & $1(2.0)$ & 0.79 & $(0.10-6.05)$ & 0.820 & $1(0.8)$ & 0.22 & $(0.03-1.59)$ & 0.133 \\
\hline CKCS & $0(0.0)$ & - & - & - & $2(4.0)$ & 1.21 & $(0.27-5.36)$ & 0.805 & $2(1.6)$ & 0.29 & $(0.07-1.20)$ & 0.087 \\
\hline Chihuahua & $1(1.39)$ & 0.09 & $(0.01-0.65)$ & 0.017 & $1(2.0)$ & 0.34 & $(0.04-2.61)$ & 0.299 & $2(1.6)$ & 0.15 & $(0.04-0.63)$ & 0.009 \\
\hline English cocker spaniel & $0(0.0)$ & - & - & - & $1(2.0)$ & 0.62 & $(0.08-4.76)$ & 0.648 & $1(0.8)$ & 0.15 & $(0.02-1.08)$ & 0.059 \\
\hline German shepherd dog & $0(0.0)$ & - & - & - & $1(2.0)$ & 0.57 & $(0.07-4.34)$ & 0.585 & $1(0.8)$ & 0.09 & $(0.01-0.64)$ & 0.016 \\
\hline Golden retriever & $7(9.7)$ & 0.64 & $(0.28-1.45)$ & 0.280 & $5(10.0)$ & 2.22 & $(2.22-1.17)$ & 0.130 & $12(9.8)$ & 1.02 & $(0.54-1.93)$ & 0.959 \\
\hline Jack russel terrier & $7(9.7)$ & 1.51 & $(0.66-3.43)$ & 0.330 & $2(4.0)$ & 0.94 & $(0.21-4.19)$ & 0.940 & $9(7.4)$ & 1.30 & $(0.63-2.67)$ & 0.473 \\
\hline Labrador retriever & $6(8.3)$ & 0.44 & $(0.18-1.05)$ & 0.065 & $7(14.0)$ & 2.85 & $(1.13-7.17)$ & 0.026 & $13(10.7)$ & 0.92 & $(0.49-1.72)$ & 0.795 \\
\hline Rottweiler & $12(16.7)$ & 2.18 & $(1.12-4.27)$ & 0.022 & $12(24.0)$ & 8.58 & $(3.89-18.91)$ & $<0.001$ & $24(19.7)$ & 3.78 & $(2.29-6.25)$ & $<0.001$ \\
\hline Shetland sheepdog & $3(4.17)$ & 0.58 & $(0.18-1.91)$ & 0.373 & $0(0.0)$ & - & - & - & $3(2.5)$ & 0.56 & $(0.17-1.81)$ & 0.332 \\
\hline Staff. bull terrier & $4(5.6)$ & 0.76 & $(0.27-2.16)$ & 0.605 & $5(10.0)$ & 2.20 & $(0.78-6.18)$ & 0.136 & $9(7.4)$ & 1.18 & $(0.58-2.42)$ & 0.651 \\
\hline SLU & $72(50.3)$ & & & & & & & & $72(27.7)$ & 1.00 & Ref. & - \\
\hline NMBU & & & & & $50(42.7)$ & & & & $50(19.2)$ & 0.65 & $(0.45-0.94)$ & 0.023 \\
\hline Other breeds & $71(49.7)$ & & & & $67(57.3)$ & & & & $138(53.1)$ & & & \\
\hline Total & $143(100.0)$ & & & & $117(100.0)$ & & & & $260(100.0)$ & & & \\
\hline
\end{tabular}

c) Medial patellar luxation

\begin{tabular}{|c|c|c|c|c|c|c|c|c|c|c|c|c|}
\hline \multirow[t]{2}{*}{ Breed } & \multicolumn{4}{|l|}{ SLU } & \multicolumn{4}{|l|}{ NMBU } & \multicolumn{4}{|l|}{ Combined } \\
\hline & N (\%) & OR & $95 \% \mathrm{Cl}$ & $\mathbf{P}$ & N (\%) & OR & $95 \% \mathrm{Cl}$ & $\mathbf{P}$ & N (\%) & OR & $95 \% \mathrm{Cl}$ & $\mathbf{P}$ \\
\hline Mixed-breed & $13(50.0)$ & 1.00 & Ref. & - & $8(38.1)$ & 1.00 & Ref. & - & $21(44.7)$ & 1.00 & Ref. & - \\
\hline CKCS & $1(3.9)$ & 0.44 & $(0.06-3.33)$ & 0.423 & $3(14.3)$ & 2.94 & $(0.78-11.11)$ & 0.112 & $4(8.2)$ & 1.25 & $(0.43-3.66)$ & 0.681 \\
\hline Chihuahua & $9(34.6)$ & 1.96 & $(0.83-4.59)$ & 0.123 & $8(38.1)$ & 4.43 & $(1.66-11.82)$ & 0.003 & $17(36.2)$ & 2.8 & $(1.47-5.32)$ & 0.002 \\
\hline Jack russel terrier & $1(3.9)$ & 0.53 & $(0.07-4.06)$ & 0.541 & $0(0.0)$ & - & - & - & $1(2.1)$ & 0.31 & $(0.42-2.30)$ & 0.252 \\
\hline Shetland sheepdog & $2(7.7)$ & 0.96 & $(0.22-4.25)$ & 0.953 & $0(0.0)$ & - & - & - & $2(4.3)$ & 0.81 & $(0.19-3.49)$ & 0.782 \\
\hline Staff. bull terrier & $0(0.0)$ & - & - & - & $2(9.5)$ & 1.43 & $(0.30-6.74)$ & 0.652 & $2(4.3)$ & 0.56 & $(0.13-2.40)$ & 0.435 \\
\hline SLU & $26(36.1)$ & & & & & & & & $26(19.8)$ & 1.00 & Ref. & - \\
\hline NMBU & & & & & $21(35.6)$ & & & & $21(16.0)$ & 0.72 & $(0.40-1.29)$ & 0.272 \\
\hline Other breeds & $46(63.9)$ & & & & $38(64.4)$ & & & & $84(64.1)$ & & & \\
\hline Total & $72(100.0)$ & & & & $59(100.0)$ & & & & $131(100.0)$ & & & \\
\hline \multicolumn{13}{|c|}{ d) Fractures of the radius and ulna } \\
\hline \multirow[t]{2}{*}{ Breed } & \multicolumn{4}{|l|}{ SLU } & \multicolumn{4}{|l|}{ NMBU } & \multicolumn{4}{|l|}{ Combined } \\
\hline & N (\%) & OR & $95 \% \mathrm{Cl}$ & $\mathbf{P}$ & N (\%) & OR & $95 \% \mathrm{Cl}$ & $\mathbf{P}$ & N (\%) & OR & $95 \% \mathrm{Cl}$ & $\mathbf{P}$ \\
\hline Mixed-breed & 19 (82.6) & 1.00 & Ref. & - & $9(52.9)$ & 1.00 & Ref. & - & $28(70.0)$ & 1.00 & Ref. & - \\
\hline$\overline{C K C S}$ & $1(4.4)$ & 0.30 & $(0.04-2.23)$ & 0.238 & $0(0.0)$ & - & - & - & $1(2.5)$ & 0.23 & $(0.31-1.71)$ & 0.151 \\
\hline Chihuahua & $3(13.0)$ & 0.45 & $(0.13-1.51)$ & 0.195 & $6(35.3)$ & 2.95 & $(1.05-8.31)$ & 0.041 & $9(22.5)$ & 1.09 & $(0.51-2.32)$ & 0.822 \\
\hline Shetland sheepdog & $0(0.0)$ & - & - & - & $2(11.8)$ & 3.04 & $(0.65-14.11)$ & 0.156 & $2(5.0)$ & 0.59 & $(0.14-2.51)$ & 0.478 \\
\hline SLU & $23(48.9)$ & & & & & & & & $23(20.2)$ & 1.00 & Ref. & - \\
\hline NMBU & & & & & $17(25.4)$ & & & & $17(14.9)$ & 0.62 & $(0.33-1.16)$ & 0.132 \\
\hline Other breeds & $24(51.1)$ & & & & $50(74.6)$ & & & & $74(64.9)$ & & & \\
\hline Total & $47(100.0)$ & & & & $67(100.0)$ & & & & $114(100.0)$ & & & \\
\hline
\end{tabular}

Results from county-stratified and combined logistic regression analyses presented as Odds ratios (OR) and 95\% confidence intervals (Cls). Breeds without cases of the disease in question were omitted

CKCS Cavalier king Charles spaniel, SLU Swedish University of Agricultural Sciences, NMBU Norwegian University of Life Sciences, ref referent category 


\section{Supplementary information}

Supplementary information accompanies this paper at https://doi. org/10.1186/s13028-020-0507-8.

Additional file 1. Control population calculations and raw registration numbers.

\section{Author details}

${ }^{1}$ Department of Companion Animal Clinical Sciences, Faculty of Veterinary Medicine, Norwegian University of Life Sciences, P.O. Box 369 sentrum, 0102 Oslo, Norway. ${ }^{2}$ Department of Clinical Sciences, Swedish University of Agricultural Sciences, P.O. Box 7054, 75007 Uppsala, Sweden. ${ }^{3}$ Department of Food Safety and Infection Biology, Faculty of Veterinary Medicine, Norwegian University of Life Sciences, P.O. Box 369 sentrum, 0102 Oslo, Norway.

Published online: 19 February 2020

\section{Reference}

1. Boge GS, Moldal ER, Dimopoulou M, Skjerve E, Bergström A. Breed susceptibility for common surgically treated orthopaedic diseases in 12 dog breeds. Acta Vet Scand. 2019;61:19. https://doi.org/10.1186/s1302 8-019-0454-4.

\section{Publisher's Note}

Springer Nature remains neutral with regard to jurisdictional claims in published maps and institutional affiliations. 